\title{
Constitutional supercooling in heavily As-doped Czochralski Si crystal
}

\section{growth}

Toshinori Taishi ${ }^{1}$, Yutaka Ohno ${ }^{2}$, Ichiro Yonenaga ${ }^{2}$

${ }^{1}$ Faculty of Engineering, Shinshu University, Wakasato, Nagano 380-8553, Japan

${ }^{2}$ Institute for Materials Research, Tohoku University, Katahira, Aoba-ku, Sendai, 980-8557, Japan

Heavily arsenic (As) doped Si crystals were grown by the Czochralski (CZ) method, and constitutional supercooling in As-doped CZ-Si crystal growth was investigated. When the As concentration in the crystal was high, cellular growth was induced and $\mathrm{SiAs}$ precipitates were then observed following the cellular structure. The As concentration increases in the cellular structure along the growth direction and around the precipitates it reaches approximately 4 at $\%$, which corresponds to the maximum solid solubility of As in Si. According to the estimation of critical growth conditions for constitutional supercooling, it qualitatively obeys the theoretical equation of constitutional supercooling. 
Keyword: A1: Doping, A1: Segregation, A1: Constitutional supercooling, A2:

Czochralski method, B1: Semiconducting silicon 


\section{Introduction}

Czochralski (CZ) -grown silicon ( $\mathrm{Si}$ ) crystals heavily doped with arsenic (As) are widely used as the substrate for epitaxial wafers, power devices and discrete devices. A low resistivity of heavily As-doped $\mathrm{Si}$ wafer has been reported as $2.7 \mathrm{~m} \Omega$-cm, corresponding to an As concentration of $1 \times 10^{19} \mathrm{~cm}^{-3}$ in the wafer [1]. A heavily As-doped Si layer is also used for the drain or source in CMOS devices. The As concentration in such a layer is $10^{20}-10^{21} \mathrm{~cm}^{-3}[2]$.

According to the phase diagram of Si and As [3], the maximum solid solubility of As in $\mathrm{Si}$ is reported to be 4 at $\%$, corresponding to $2.2 \times 10^{21} \mathrm{~cm}^{-3}$. When the As content in a liquid is higher than 40 at \%, SiAs compound crystallizes with Si solid with the As content of the maximum solid solubility [3]. Generally, in heavily doped crystal growth, when the impurity concentration increases before reaching the maximum solid solubility, cellular growth due to constitutional supercooling occurs and this finally leads to a polycrystalline structure. In the case of $\mathrm{Si}$, constitutional supercooling in $\mathrm{CZ}$ crystal growth heavily doped with $\mathrm{Sb}$ [4], B [5] and $\mathrm{Ge}$ [6] has been reported. The discrimination of constitutional supercooling has been theoretically proposed as follows [7]:

$$
\frac{G}{V}<\frac{m C_{0}}{D} \frac{1-k}{k}
$$


where $G$ is temperature gradient in the melt just below the crystal/melt interface, $R$ is the growth rate of the crystal, $m$ is the slope of the liquidus line, $k$ is the equilibrium segregation coefficient, $C_{0}$ is the impurity concentration in the melt and $D$ is the diffusion coefficient of the impurity in the melt. Constitutional supercooling occurs readily when the segregation coefficient is small and the growth rate is large. Indeed, the equilibrium segregation coefficients of $\mathrm{Sb}, \mathrm{B}$ and $\mathrm{Ge}$ are 0.023 [8], 0.8 [8] and 0.5 [6], respectively. In the case of $\mathrm{B}, k_{0}$ has been found to decrease with increasing $\mathrm{B}$ concentration in the melt, to the value 0.38 when constitutional supercooling occurs [5]. The concentrations of $\mathrm{B}$ and $\mathrm{Ge}$ in $\mathrm{Si}$ crystals grown at a pulling rate of approximately $1 \mathrm{~mm} / \mathrm{min}$ when constitutional supercooling occurs were reported to be $2.8 \times 10^{20}[5]$ and, $5.7 \times 10^{20} \mathrm{~cm}^{-3}[6]$, respectively. Such concentrations are lower than the maximum solid solubilities of each impurity.

In the present study, heavily As-doped Si crystals were grown by the $\mathrm{CZ}$ method. A cellular structure, where constitutional supercooling occurs, and precipitation of SiAs following the cellular structure were exhibited. The occurrence of constitutional supercooling in As-doped CZ-Si crystal growth is discussed theoretically.

\section{Experimental procedure}


An As-containing $\mathrm{Si}$ ingot was prepared by adding As to the $\mathrm{Si}$ melt with subsequent quenching to room temperature. The As concentration in the ingot was approximately $1.0 \times 10^{20} \mathrm{~cm}^{-3}$. A silica crucible 2 in. in diameter was charged with these ingots, together with high purity Si raw materials $(300 \Omega-\mathrm{cm})$. Considering that the equilibrium segregation coefficient of $\mathrm{As}$ in $\mathrm{Si}$ is 0.3 [9], the As concentration in the top region of the Si crystals was controlled to be $2.0 \times 10^{19} \mathrm{~cm}^{-3}$. The silica crucible was set inside a graphite heater $70 \mathrm{~mm}$ in diameter surrounded by graphite heat shields in a stainless chamber. After evacuating the chamber, Ar gas was filled into the chamber and the pressure was controlled to be 1 atm. A Si single crystal in the [001] orientation with a size of $4.5 \times 4.5 \times 50 \mathrm{~mm}^{3}$ was used as a seed. As-doped Si crystals were grown at a pulling rate of $20 \mathrm{~mm} / \mathrm{h}$. The crystal rotational rate was $6 \mathrm{rpm}$ and the crucible rotational rate was $1.8 \mathrm{rpm}$ in the opposite direction. After the growth, the crystal was cooled to room temperature over a period of $5 \mathrm{~h}$.

The grown crystals were cut into wafers $1 \mathrm{~mm}$ thick. The wafers were ground and polished, and were observed by optical microscopy after the application of Dash etch (a mixture of $\mathrm{HNO}_{3}$, $\mathrm{HF}$ and $\mathrm{CH}_{3} \mathrm{COOH}$ in a 4:1:10 ratio) for 10 min at room temperature. The As concentrations in some specimens were measured quantitatively by electron probe microanalysis (EPMA) using JXA-8621MX (JEOL). Square specimens with 
dimensions of $5 \times 5 \times 1.5 \mathrm{~mm}^{3}$ were prepared from the grown crystal, and Hall coefficient and resistance measurements using the Vander Pauw method were conducted at room temperature (RT) to determine the variation in the donor concentration caused by the As atoms.

\section{Results and discussion}

Figure 1 shows a typical As-doped CZ-Si boule grown at a pulling rate of $20 \mathrm{~mm} / \mathrm{h}$. The As concentration at the top of the crystal was $2 \times 10^{19} \mathrm{~cm}^{-3}$. Four habit lines appeared at the shoulder and in the main body of the crystal, indicating that a Si single crystal was obtained. However, they disappeared at the position indicated by a white arrow in Fig. 1, where the solidified fraction corresponded to 0.86 . The As concentration at that position in the crystal was $1 \times 10^{20} \mathrm{~cm}^{-3}$.

Figure 2 (a) shows an optical microscopic image of an As-doped Si wafer cut horizontally after the preferential etching. Many rectangular shaped structures can be observed near the periphery in the wafer. These patterns are a typical cellular structure, indicating that constitutional supercooling occurs during the crystal growth. Another optical microscopic image of an As-doped Si wafer cut vertically along the growth direction is shown in Fig. 2 (b). The growth interface was convex towards the crystal 
top in the main body. However, below this the interface shape became zigzag and unstable, and cellular structures appeared near the periphery. Further down, many black precipitates following the cellular structure may be observed, and then the crystal led to a polycrystalline form. These results therefore indicate that polycrystallization originates from constitutional supercooling.

Figure 3 (a) shows a back-scattered image of the wafer cut vertically through the area that includes both cellular structure and precipitate. The image of the precipitate is brighter than that of matrix, indicating that the molecular weight of the precipitate is larger than that in the Si matrix. Figure 3 (b) shows the relationship between the vertical distance from the precipitate and the As content in the crystal. In the cellular structure (labeled as line 1 in Fig. 3 (a)), the As content in the Si crystal increases with closeness to the precipitates and finally reached 50 at $\%$ on the precipitate, although remarkable increase was not detected on the left side (labeled as line 2 in Fig. 3 (a)) $80 \mu \mathrm{m}$ from the line 1. These indicate that the precipitate is SiAs compound. In addition, just above the SiAs precipitate, the As content was approximately 4 at\%. This value corresponds well to the maximum solid solubility of As in $\mathrm{Si}$ [3]. According to similar analyses around other precipitates, many regions with an As content of 4 at\% just above the SiAs precipitate were observed. 
Based on these results, a model of interface shape, precipitation and polyscrystallization process is considered, as shown in Fig. 4. At first, when the As concentration in the Si crystal increases, the shape of the growth interface becomes unstable (Fig. 4 (a)) and As-rich columns are formed by constitutional supercooling [10]. Cellular structures are then generated with facet growth due to continuation of constitutional supercooling (Fig. 4 (b)) [5]. Here, in an As-rich column, the solidification delay from the matrix, then As atoms furthermore increase in the column. When the As content reaches 40 at $\%$ in the column, two kinds of components are solidified [3], one is As-enriched $\mathrm{Si}$ with an As content of 4 at\%, and the other is $\mathrm{SiAs}$ precipitates. The As-enriched Si regions exist just above or around the SiAs precipitates (Fig. 4 (c)). Line 1 in Fig. 3 (a) is one such column. These solidification processes correspond to the phase diagram of Si and As [3]. Finally, polycrystallization occurs due to constitutional supercooling and SiAs precipitates.

Critical growth conditions for the occurrence of constitutional supercooling were estimated using eq. (1). In the present case, the physical parameters are follows: $k=0.3$ [9], $D=3.3 \times 10^{-4} \mathrm{~cm}^{-2}$ [11], and $m=7 \times 10^{-21}{ }^{\circ} \mathrm{Ccm}^{3}$ [3]. The growth conditions are follows: $G=15^{\circ} \mathrm{C} / \mathrm{cm} \mathrm{[5],} R=0.0055 \mathrm{~mm} / \mathrm{s}$ and $C_{0}=C_{\mathrm{S}} / k=3.3 \times 10^{20} \mathrm{~cm}^{-3}$. As results of estimation for critical growth conditions, the left hand side of eq. (1) was smaller 
than the right hand side, indicating that the occurrence of constitutional supercooling is consistent with the theory. Further analysis of constitutional supercooling in heavily As-doped Si crystal growth, especially under different growth conditions such as growth rate and As concentration in the crystals, will be reported elsewhere.

\section{Conclusions}

Heavily As-doped Si crystals were grown by the CZ method, and constitutional supercooling in As-doped CZ-Si crystal growth was investigated. When the As concentration in the crystal was $1 \times 10^{20} \mathrm{~cm}^{-3}$, cellular growth was induced in the crystal and SiAs precipitates bellow the cellular structure were observed. As the concentration increases in the cellular structure along the growth direction and around the precipitates it reaches approximately 4 at\%, which corresponds to the solid solubility of As in Si. A model of the interface shape, SiAs precipitation and polyscrystallization process has been considered, based on the phase diagram of Si and As. Constitutional supercooling occurs when the pulling rate is high and when the As concentration in the crystal is high, critical growth conditions qualitatively consistent in the theoretical equation of constitutional supercooling. 


\section{Acknowledgements}

The authors thank Mr. M. Ohwa, Dr. K. Kashima and Mr. S. Narimatsu of Covalent Materials Corporation, Mr. Y. Murakami of Tohoku University and Prof. K. Hoshikawa of Shinshu University for their kind support. This work was performed under the Inter-university Cooperative Research Program of the Institute for Materials Research, Tohoku University. 


\section{References}

[1] B. Wang, X. Zhang, X. Ma, D. Yang, Effects of high temperature rapid thermal processing on oxygen precipitation in heavily arsenic-doped Czochralski Silicon, Journal of Crystal Growth 318 (2011) 183-186.

[2] G. H. Lu, Q. Wang, F. Liu, The role of vacancy on trapping interstitial O in heavily As-doped Si, Applied Physics Letters 92 (2008) 211906(1-3).

[3] R. W. Olesinski, G. J. Abbaschian, The As-Si (Arsenic-Silicon) system, Bulletin of Alloy Phase Diagrams 6 (1985) 254-258.

[4] K.M. Kim, Interface Morphological Instability in Czochralski silicon crystal growth from heavily Sb-doped melt, Journal of Electrochemical Society 126 (1979) 875.

[5] T. Taishi, X. Huang, M. Kubota, T. Kajigaya, T. Fukami, K. Hoshikawa, Heavily boron-doped silicon single crystal growth: Constitutional supercooling, Japanese Journal of Applied Physics 39 (2000) L5-L8.

[6] T. Taishi, X. Huang, I. Yonenaga, K. Hoshikawa, Dislocation behavior in heavily germanium-doped silicon crystal, Materials Science and Semiconductor Processing 5 (2003) 409-412.

[7] W. A. Tiller, K. A. Jackson, J. W. Rutter, B. Chalmers, The redistribution of solute atoms during the solidification of melts, Acta Metallurgia 1 (1953) 428-437. 
[8] F. A. Trumbore, Solid solubilities of impurity elements in germanium and silicon, The Bell System Technical Journal 39 (1960) 205-233.

[9] J. A. Burton, Impurity centers in Ge and Si, Physica 20 (1954) 845-854.

[10] J. W. Rutter, B. Chalmers, A prismatic substructure formed during solidification of metals, Canadian Journal of Physics 31 (1953) 15-39.

[11] H. Kodera, Diffusion coefficient of impurities in silicon melt, Japanese Journal of Applied Physics 2 (1963) 212-219. 


\section{Figure captions}

Fig. 1 Photograph of heavily As-doped CZ-Si boule with an As concentration of $2 \mathrm{x}$ $10^{19} \mathrm{~cm}^{-3}$

Fig. 2 Photograph of heavily As-doped wafer where the cellular growth has been induced.

Fig. 3 (a) Back-scattered image of a SiAs precipitate below the cellular structure, (b)

As concentrations on line 1 and line 2 in the Si wafer analyzed be EPMA.

Fig. 4 Illustrations of interface shape and precipitation process after constitutional supercooling occurs in heavily As-doped CZ-Si crystal growth. (a) Unstable growth interface appears, then (b) cellular growth appears due to constitutional supercooling, finally (c) As-rich columns is formed and SiAs precipitates via solidification of As-enriched $\mathrm{Si}$ with an As content of 4 at\%, corresponding to the maximum solid solubility of As in Si. 


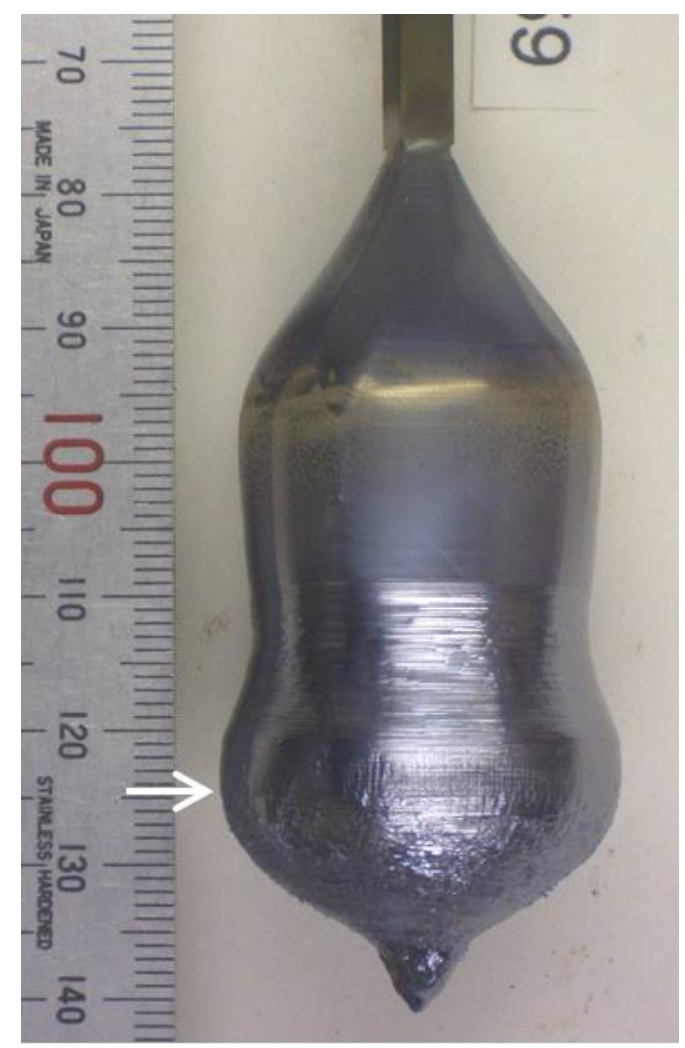

Fig. 1 

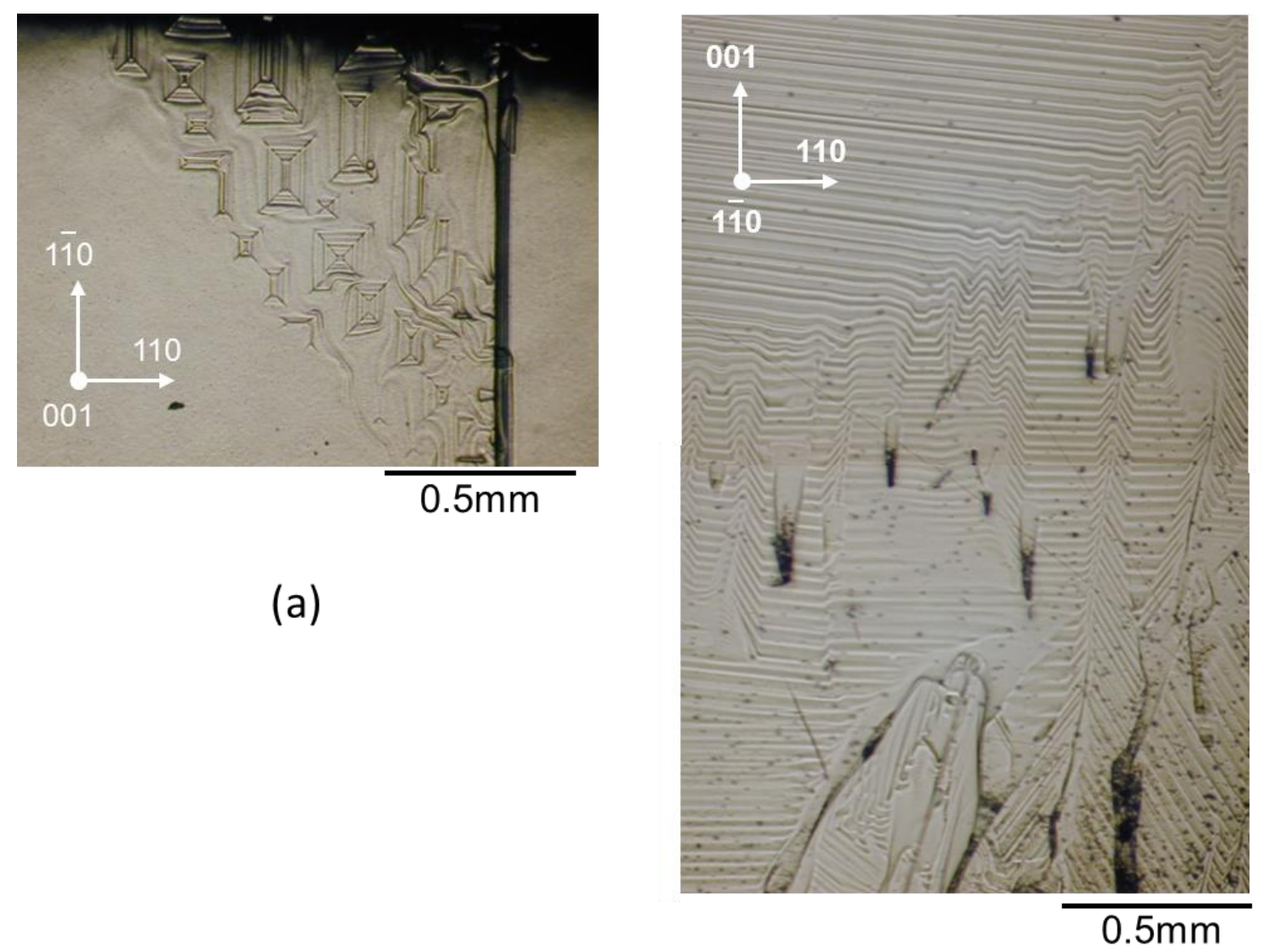

(a)

(b)

Fig. 2 
line 2 line 1

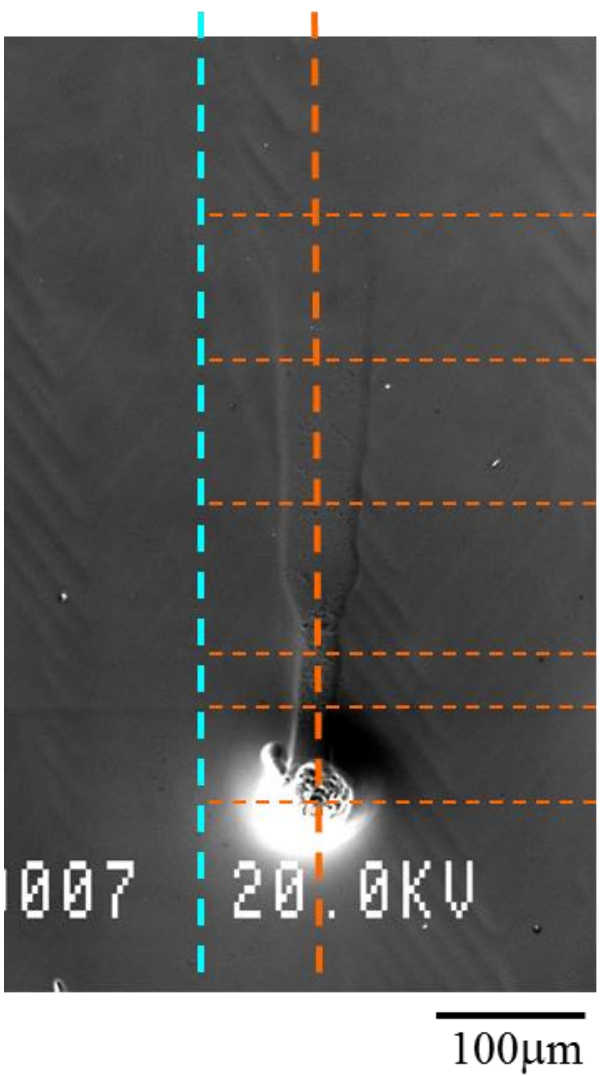

(a)

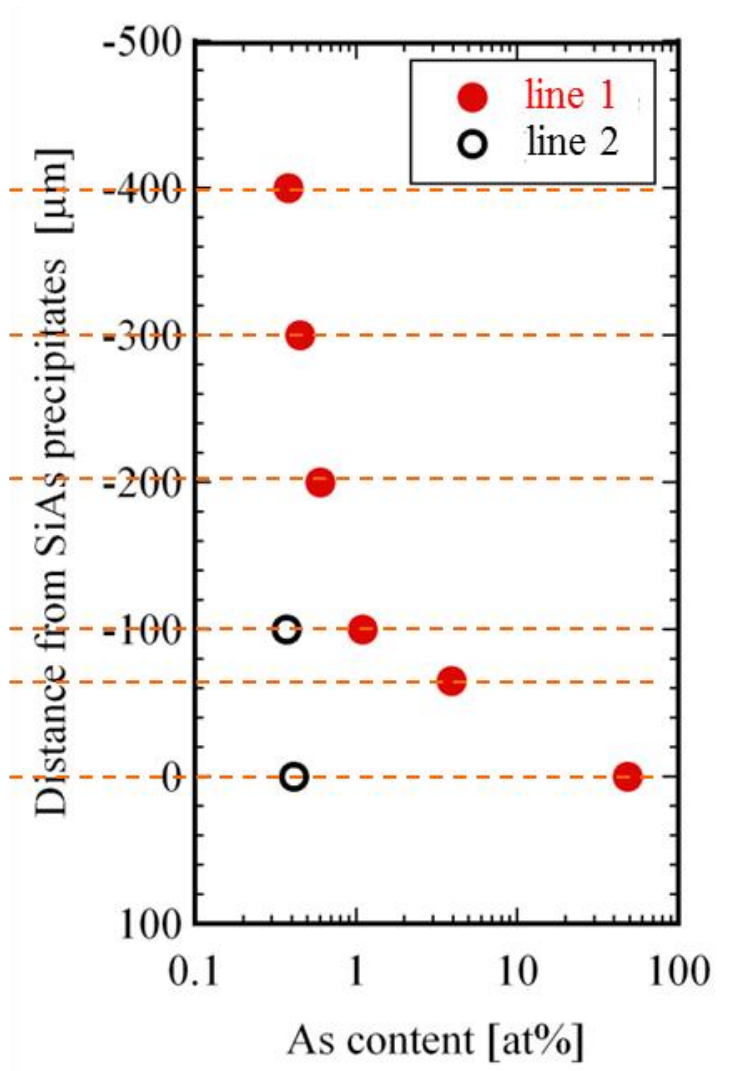

(b)

Fig. 3 


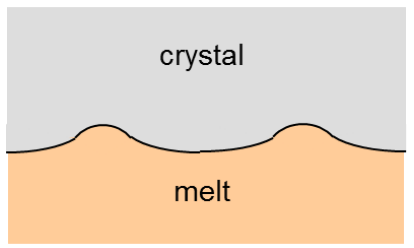

(a)

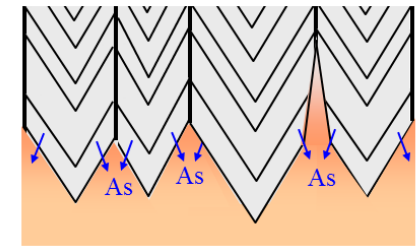

(b)

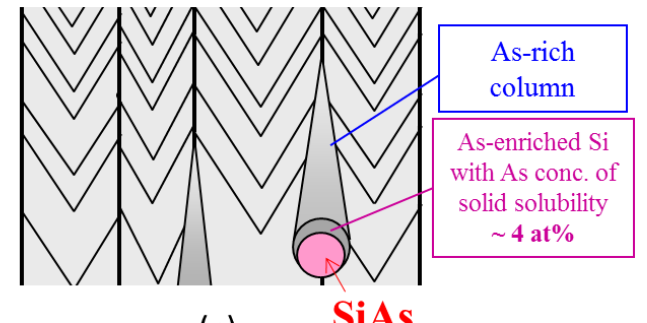

(c)

Fig. 4 